\title{
Solitonics with Polyacetylenes
}

\author{
Daniel Hernangómez-Pérez,* Suman Gunasekaran, Latha Venkataraman, and Ferdinand Evers*
}

Cite This: Nano Lett. 2020, 20, 2615-2619

Read Online

ABSTRACT: Polyacetylene molecular wires have attracted a longstanding interest for the past 40 years. From a fundamental perspective, there are two main reasons for the interest. First, polyacetylenes are a prime realization of a one-dimensional topological insulator. Second, long molecules support freely propagating topological domain-wall states, so-called "solitons," which provide an early paradigm for spin-charge separation.

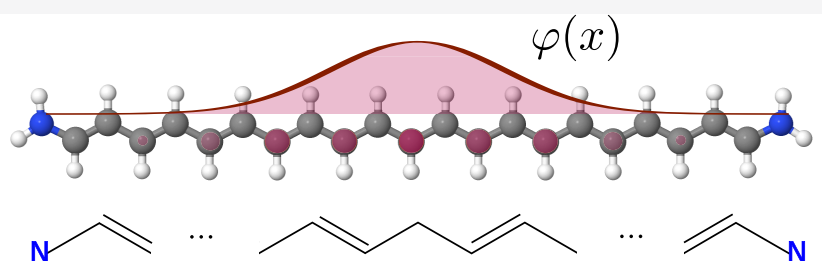
Because of recent experimental developments, individual polyacetylene chains can now be synthesized on substrates. Motivated by this breakthrough, we here propose a novel way for chemically supported soliton design in these systems. We demonstrate how to control the soliton position and how to read it out via external means. Also, we show how extra soliton-antisoliton pairs arise when applying a moderate static electric field. We thus make a step toward functionality of electronic devices based on soliton manipulation, that is, "solitonics".

KEYWORDS: polyacetylene, solitons, density functional theory, topology

- ver since the seminal works by $\mathrm{Su}$, Schrieffer, Heeger (SSH) and Kivelson, ${ }^{1-3}$ (trans-)polyacetylene $\left(\mathrm{C}_{2} \mathrm{H}_{2}\right) n$ (PA) has been extensively studied. At present, there is a revived interest in this polymeric species because arguably these molecules represent the simplest realization of a topological insulator. Correspondingly, the simplified $\mathrm{SSH}$ model is textbook material and the associated physics constitutes the introductory part of many lectures on topological physics. ${ }^{4}$

Interestingly, a most intriguing phenomenon featured by PA has a topological origin, which bears similarities to a frequently investigated material class, the topological insulators. The molecule exhibits a doubly degenerate ground state that allows for the formation of a domain wall with an associated topological boundary state, the so-called "soliton", see Figure 1. Its interesting property is that it can almost freely propagate; it also represents an early example for exotic excitations that exhibits spin-charge separation. ${ }^{1,2,5,6}$

Even though the relevance of the system as a paradigm for topology and strong correlations in physics has been acknowledged more than 30 years ago, solitonic excitations in PA chains have remained relatively unexplored experimentally and not so well understood. Investigations took place mostly in polymers, where indirect evidence for solitons can be seen in optical excitations ${ }^{7-10}$ and recently also in momentumspace using a chain of cold atoms. ${ }^{11}$ It was only very recently that an on-surface synthesis and investigations of individual PA chains became feasible. ${ }^{12}$

Our proposal is partially motivated by these breakthroughs. We here present an extensive study based on the density functional theory and demonstrate that (i) individual solitons can be built into individual PAs by choosing proper end groups, (ii) solitons can be moved by the application of a weak static electric field, and (iii) the position of the soliton impacts the conductance of the finite wire; reciprocally, the position can be reconstructed from the $\mathrm{d} I / \mathrm{d} V$-characteristics. Thus, the essential ingredients of a soliton-based technology, for example, a transistor, are here represented. Finally, we show that at slightly larger but still moderate field strength that even extra soliton-antisoliton pairs can appear giving rise to a stable string of topological domain-walls.

Before explaining our main ideas and results, we set the stage and briefly recapitulate basic properties of solitons in PA. The soliton resides at the boundary between two domains of a PA wire. In each domain, there is a spectral gap around the Fermi energy, that is, each is a band insulator. ${ }^{13}$ The gap is brought about by the Peierls' effect which manifests structurally in the form of bond-length alternation (BLA), that is, the difference in neighboring bond lengths; see Figure 1a. As it is typical of topological systems, the domain wall supports a boundary state with an energy situated in the middle of the bulk gap of either neighboring insulating segment. For PA wires without extra charge, $Q=0$, this state is singly occupied and carries a spin $S$ $=1 / 2$; see Figure $1 \mathrm{a}$. When adding an extra electron to the molecule, $Q=-|e|$, the boundary state is doubly occupied and becomes a soliton with $S=0$.

Received: January 11, 2020

Revised: February 29, 2020

Published: March 3, 2020 
$(a)$<smiles>C/C=C/[CH]/C=C/[13CH]=CN</smiles>

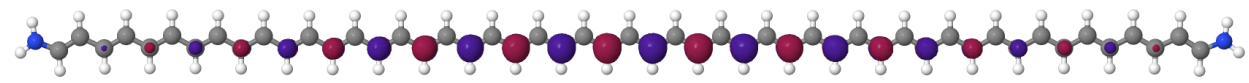

Figure 1. Chemical structure of polymethine molecules considered in this work. (a) The resonant configuration for an uncharged neutral $(Q=0)$ molecule is shown. The BLA is represented by the sequence of single and double bonds. Where BLA changes sign a domain-wall can form. (b) Corresponding Kohn-Sham orbital that forms the topologically protected domain-wall state in a wire of length $L=63$ "sites".
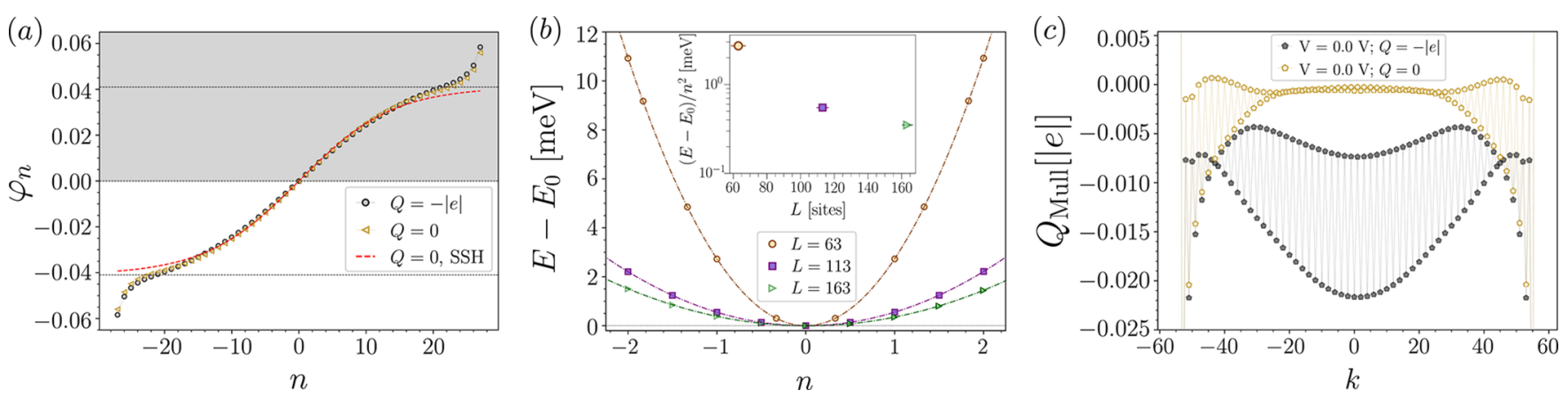

Figure 2. (a) Order parameter $\varphi_{n}$ as defined in eq 1 plotted along the PA for the charged (circles) and uncharged (triangles) species. The length is measured in terms of unit cells ("sites") with the center cell situated around the inversion center and taken as $n=0$; the total length is 55 unit cells, which correspond to 113 molecular backbone atoms or $137.52 \AA$. Gray and white backgrounds indicate the two phases discriminated by the sign of $\varphi_{n}$; the dotted horizontal lines represent the order parameter as would be obtained in the infinite wire. The dashed red line shows the shape of the order parameter predicted by the SSH model with a correlation length of $\widetilde{\xi} \simeq 14$ unit cells or $34.44 \AA$ (with lattice unit vector $a=2.46 \AA$ as obtained from ab initio calculations). (b) Confinement potential (units $\mathrm{meV}$ ) of the soliton for three different polymethine wires of lengths $L=63$, 113, 163 "sites" near the potential bottom. As in (a), the length is measured in terms of unit cells, $n$. Inset: Curvature $\left(E-E_{0}\right) / n^{2}$. The logarithmic scale emphasizes its rapid decrease with increasing system size $L$. (c) Charge distribution (Mulliken) of the neutral $(Q=0$, gold) and charged $(Q=$ $-\mid e l$, black) PA with $L=113$; the coordinate $k$ denotes the site index along the molecular backbone here used to highlight even-odd oscillations expected from Figure $1 \mathrm{~b}$.

The first computational result we report here is that the existence of solitons can be controlled through chemical design (see computational details in the Supporting Information). In Figure 1, a corresponding example is shown. The design principle is based on choosing the end groups, such as aminogroups, shown in Figure 1, or methyl- and methylene-groups, shown in the Supporting Information; they control the BLA pattern in the wire. In Figure 1, the formation of a domain wall was further enforced by implementing a mirror symmetry, which is possible for odd-numbered chains.

A prerequisite for the formation of the domain wall and hence the soliton is BLA; it is fully developed only in wires longer than a critical number of unit cells, $N_{c}$. With the amine end groups, our DFT calculations suggest that $N_{c} \simeq 20$. Shorter wires have structurally dictated the domain wall as discussed in the Supporting Information. The shape of the domain-wall is shown in Figure 2a for a wire that contains 55 unit cells (corresponding to 113 molecular backbone atoms). It is quantified in terms of the normalized BLA (order parameter) defined as

$$
\varphi_{n}=\frac{l_{\mathrm{oe}}^{(n)}-l_{\mathrm{eo}}^{(n)}}{\left(l_{\mathrm{oe}}^{(n)}+l_{\mathrm{eo}}^{(n)}\right) / 2}
$$

where $l_{\mathrm{oe}}^{(n)}\left(l_{\mathrm{eo}}^{(n)}\right)$ is the bond length between the odd-numbered and the even numbered (even-numbered and odd-numbered) atom in the $n$th unit cell. In the long-wire limit, we have $\varphi_{\infty}=$ $\lim _{n \rightarrow \infty} \varphi_{n} \simeq 4 \%$. The functional form of $\varphi_{n}$ is expected to approach $\varphi_{\infty} \tanh (n / \xi),{ }^{1}$ as represented by the red dashed line in Figure $2 \mathrm{a}$. The parameter $\xi \simeq 14$ corresponds to the width of the domain wall, that is, the size of the soliton.

We show in Figure 2a that the shape of the domain wall is only very weakly sensistive to the charge it carries, $Q$. This is important because, as we will see, only charged walls can be moved by electric fields. Indeed, performing a nonlinear fit we find that $\xi$ is similar for both cases: $\widetilde{\xi}=12.8 \pm 0.5$ and $\widetilde{\xi}=12.8 \pm 0.7$ for $Q=0$ and $Q=-|e|$, respectively. These values are consistent with earlier measurements ${ }^{1,14,15}$ but roughly $50 \%$ larger than previous results from the model ${ }^{16}$ or semiempirical calculations. ${ }^{17}$

The position of the domain-wall is in the center of the polymethine wire because of the molecular mirror symmetry. The corresponding confinement potential is shown in Figure $2 \mathrm{~b}$. Crucially, the potential curvature diminishes very rapidly with increasing length of the wire. Thus, the force required to shift the domain wall is small for long enough wires and so the soliton can easily be moved. The size-dependency of the curvature reflects the rapidly decreasing overlap of the soliton tails with the edge of the wire (see inset with logarithmic representation of the curvature of the potential). Indeed, a soliton in polymethine represents a kind of "particle in a box" experiencing a confinement potential that is nearly flat in the center and that reflects the shape of the soliton near the confining edges. 
(a)

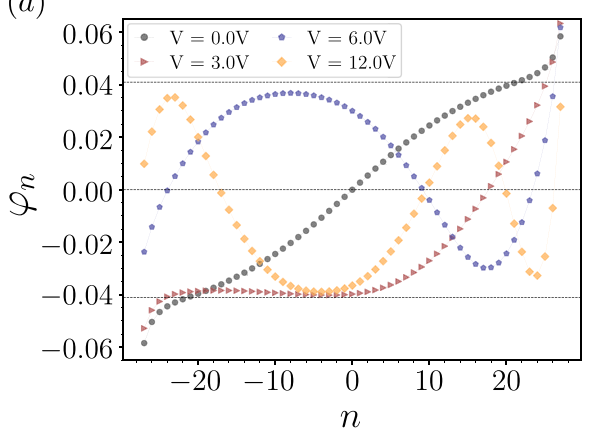

(b)

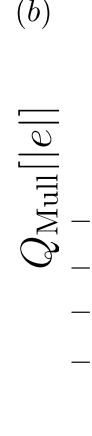

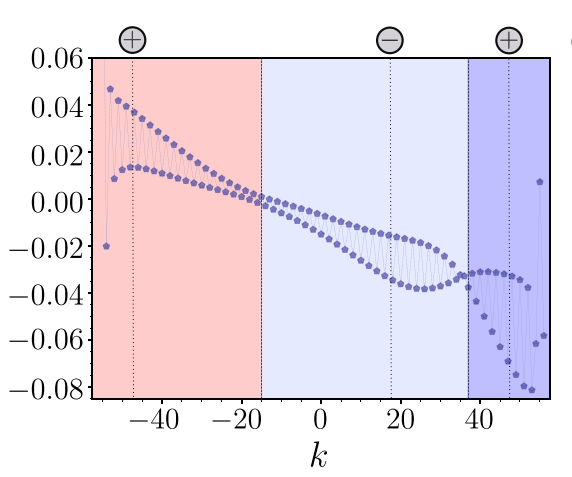

$(c)$

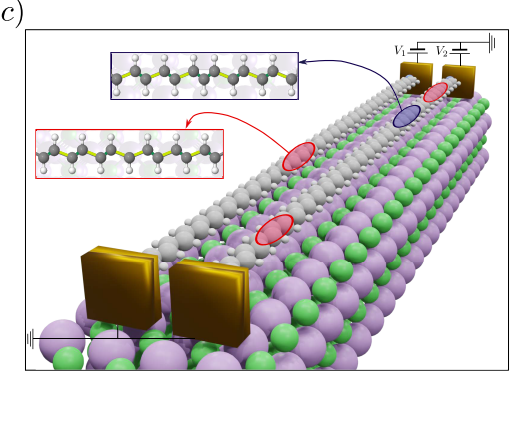

Figure 3. (a) Evolution of the order parameter, that is, the BLA, of a charged polymethine wire $(Q=-l e l, L=113$, that is, 55 unit cells $)$ with increasing longitudinal voltage $V$. At weak fields, about $50 \mathrm{mV}$ per unit cell (dark red triangles), the domain wall is pushed close to one of the edges of the wire. For fields reaching $\sim 100 \mathrm{mV}$ per unit cell (dark blue), an island forms in the range $-25 \lesssim n \lesssim 9$ and correspondingly an extra pair of domain walls appears. At higher fields, for example, $200 \mathrm{mV}$ per unit cell, a second island has emerged contributing one more pair of domain walls. At even higher fields, the process proliferates. (b) Charge distribution (Mulliken of functional unit $\mathrm{CH}$ ) of the polymethine wire with an excess charge $Q=-|e|$ at $V=6 \mathrm{~V}$, that is, after pair production. The center of each domain wall, as obtained from panel a, is indicated by the upper gray circles, with + denoting the soliton and - denoting the antisoliton. The charge content per domain wall is nearly \pm lel as estimated by summing up all the Mulliken charge per colored box (left soliton, 0.88lel; center (antisoliton), -0.92lel; right soliton, $-0.96|e|)$. (c) Illustration of two PA wires that carry solitons (red spots) and antisolitons (black spot). The electrodes (brown squares) may be used for soliton shifting and/or pair creation, but they also can serve to monitor the number and the position of the solitons by means of a conductance measurement.

Unlike what one would perhaps expect from the shape of the electronic orbital displayed in Figure $1 b$, the charge density in the neutral polymethine molecule $(Q=0)$ is rather homogeneous in the vicinity of the domain wall; see Figure 2c. Therefore, the domain wall at $Q=0$ does not respond to electric fields; because the domain wall state carries a spin $S=$ $1 / 2$, in this case the soliton could be moved by applying a magnetic field gradient. This behavior is a manifestation of spin-charge separation in PA molecular wires. ${ }^{1}$ Conversely, charging the molecule with one extra electron promotes the domain-wall state, that is, $Q=-|e|$ and zero spin. The charged domain-wall state no longer responds to magnetic fields. However, since the excess charge nicely accumulates near the domain wall (Figure $2 \mathrm{c}$ ) one can drag the soliton by applying a static electric field. Hence, all-electric solitonics operates with charged solitons.

Our second set of results is summarized in Figure 3. We demonstrate that after charging the domain wall state, $Q=-|e|$, the soliton can be moved to (almost) any position along the polymethine wire by applying a moderate static electric field. As is shown in Figure 3a, at only $3 \mathrm{~V}$ along a wire of 55 units the soliton shifts through most of the wire toward the edge.

The position of the soliton after moving can be determined, in principle, via a polarization measurement ("read out"), as described in Supporting Information. With an eye on a solitonbased "on-chip technology", we here propose an alternative method based on a transport measurement. The operating principle exploits the exponential sensitivity of the differential conductance to the position of the soliton. It becomes evident after shifting away the soliton from the symmetry position, because the transport resonance couples asymmetrically to the source and drain with an exponential envelope. By monitoring the conductance suppression away from its maximum value, the soliton position can be inferred. As a practical demonstration, we have computed the transmission of the polymethine wire and analyzed how it reacts to shifting the domain wall (see Supporting Information).

Once the applied voltage $V$ has reached values so large that the soliton has been drawn to an edge $(V \simeq 2.5 \mathrm{~V}$ in Figure $3 a)$, this soliton can no longer contribute to screening if $V$ is increased further. At this voltage scale, an interesting effect sets in, that is, pair creation. It could potentially be of technological interest as it introduces a threshold scale in the $I-V$. Indeed, by tuning the voltages higher the next natural scale one would expect to occur is the bandwidth, $\Delta$. If $V$ even exceeds this scale, an electrical breakdown is expected. Intriguingly, the breakdown is preempted by the formation of a region at which the bond order parameter exhibits a sign inversion, see Figure $3 a$.

The creation of extra topological domain walls is an effective mechanism for the molecule to release electrostatic energy. One of the two new walls is an antisoliton, which can accommodate one extra charge, $-\mid e l$, and transport it in the direction of the original soliton. Note that the first soliton and the antisoliton will not annihilate one another. The process is inhibited by conservation of the Coulomb charge. The hole left behind is carried by the second soliton in the reverse direction toward the other edge, as shown in Figure 3b. Effectively, the solitons allow the molecular wire to transport charges over large distances without undergoing the electric breakdown, therefore releasing electrostatic energy.

Figure $3 \mathrm{a}$ indicates that at even larger voltages a second soliton-antisoliton pair can be created. The process will continue until eventually a maximum number of pairs fitting into the wire has been reached. At this stage, the polymethine molecule is going to host an alternating sequence of $m+1$ solitons and $m$ antisolitons that accommodate $m+1$ electrons on one side of the wire and $m$ holes on the other, regularly spaced from each other as shown in Figure 3c.

To test our theoretical predictions, the recent experimental breakthrough in on-substrate synthesis of long PA wires ${ }^{12}$ holds great promise. Specifically, chains of 50 carbon units and more in length have been synthesized, which significantly exceeds the estimate for the critical length $2 N_{\mathrm{c}} \gtrsim 40$ atoms that we obtained for the case of amino-end groups. Moreover, the authors were able to work on insulating substrates and reported first indications of a localized domain-wall state. Toward the scenario depicted in Figure $3 c$, three minor elements are still needed that hopefully with further experimental progress will be achieved: (i) the excess charge 
of the molecule needs to be controlled, which could be feasible employing experimental techniques along the lines of ref 18; (ii) symmetry-breaking end groups need to be attached, such as the amino-groups we have used in this work; and (iii) the possibility for an in-plane electric field needs to be implemented. Recalling recent experiments on insulating substrates, the latter can be realized, for example, also by controlled STM-driven charging of surface elements, such as metallic adatoms. ${ }^{18}$ Exploring the technological potential of solitonics should be promising, because several different regimes are available that can be addressed in an all-electronic setup. These regimes are expected to be experimentally feasible at room temperature since the latter is substantially smaller than the band gap.

We present two mechanisms for a soliton-induced timedependent modulation of an ac. (i) Upon application of a moderate ac-field of amplitude $E(\Omega)$ and frequency $\Omega$, an isolated soliton will oscillate with an amplitude $x_{0} \approx \mathrm{eE}(\Omega)$ / $M_{s} \Omega^{2}$. Because the soliton mass $M_{s}$ is on the scale of the electron mass, $M_{\mathrm{s}} \approx 6 m_{\mathrm{e}}{ }^{1}$ one estimates that shuttling from one electrode to the other is achieved at frequencies in the terahertz regime. Because the current is exponentially sensitive to the soliton position, one expects a conversion of an acvoltage into a current signal that is very sharply peaked in time ("bursts") indicating the instance when the shuttle passes the point of maximum transmission. (ii) When applying a stronger ac-field, soliton-antisoliton pairs can be formed. Similar to the (static) polarization (Figure 5 in Supporting Information), steplike features are also to be expected in the time-dependent current that counts the pairs which are destroyed and created new in a single voltage cycle.

Finally, as has been demonstrated by Wang et al., ${ }^{12}$ pairs of PA wires can be synthesized at distances of only a few lattice constants. This opens up a perspective to study arrays of wires, where solitons experience an electrostatic interaction with solitons on neighboring wires; see Figure $3 \mathrm{c}$ for illustration. In this situation, collective effects might be expected, such as the creation of a soliton-antisoliton pair in one wire due to the presence of a soliton in the neighboring one. Conceivably, such arrays could have critical points or even unstable phases that could act as a detector; perturbing a center soliton then would produce a cascade of extra pairs spreading all over the array.

We have presented calculations based on the density functional theory that demonstrate that PA chains with amino-based end groups possess a domain-wall (soliton) state when they exceed a length of roughly 40 carbon units. The soliton is confined by a relatively weak confinement potential and after charging it can be displaced by weak (static) electric fields. The resulting position of the soliton can be read out via a conductance measurement. Because the conductance is exponentially sensitive to the soliton position, this could create a single soliton-based transistor. At larger voltages, a string of soliton-antisoliton pairs aligns along the wire that is stabilized by strong Coulomb repulsion. The nature of the resulting state is likely strongly correlated and therefore constitutes a promising arena for further theoretical and experimental research.

\section{ASSOCIATED CONTENT}

\section{(s) Supporting Information}

The Supporting Information is available free of charge at https://pubs.acs.org/doi/10.1021/acs.nanolett.0c00136.
Methods and additional computational data (bondlength alternation in short polyacetylene molecules, bond-length alternation in polyacetylene wires with methyl and methylene end-groups, read out of the position of a single soliton and creation of new domain walls from the induced polarization, electrical read out of the position of a single soliton from quantum transport calculations) (PDF)

\section{AUTHOR INFORMATION}

\section{Corresponding Authors}

Daniel Hernangómez-Pérez - Institute of Theoretical Physics, University of Regensburg, 93040 Regensburg, Germany; - orcid.org/0000-0002-4277-0236;

Email: daniel.hernangomez@ur.de

Ferdinand Evers - Institute of Theoretical Physics, University of Regensburg, 93040 Regensburg, Germany;

Email: ferdinand.evers.de

\section{Authors}

Suman Gunasekaran - Department of Chemistry, Columbia University, New York, New York 10027, United States; (1) orcid.org/0000-0001-5974-0642

Latha Venkataraman - Department of Chemistry and Department of Applied Physics, Columbia University, New York, New York 10027, United States; 이이. orcid.org/00000002-6957-6089

Complete contact information is available at: https://pubs.acs.org/10.1021/acs.nanolett.0c00136

\section{Notes}

The authors declare no competing financial interest.

\section{ACKNOWLEDGMENTS}

D.H.-P. acknowledges M. Camarasa-Gómez, A. Donarini, M. Marganska-Lyzniak, R. Korytár, and, in particular, J. Repp for constructive discussions. Funding by the Deutsche Forschungsgemeinschaft (DFG, German Research Foundation), Project-ID 314695032, SFB 1277 (subprojects A03, B01) is acknowledged. S.G. is supported by a NSF Graduate Research Fellowship under Grant DGE-1644869. L.V. acknowledges support from the NSF Grant DMR-1807580.

\section{REFERENCES}

(1) Heeger, A. J.; Kivelson, S.; Schrieffer, J. R.; Su, W. P. Solitons in conducting polymers,. Rev. Mod. Phys. 1988, 60, 781-850.

(2) Kivelson, S. Soliton model of polyacetylene. In Solitons, Modern Problems in Condensed Matter Sciences, 1st ed.; Trullinger, S. E., Zakharov, V. E., Pokrovsky, V. L., Eds.; Elsevier Science Ltd, 1986.

(3) Barford, W. Electronic and Optical Properties of Conjugated Polymers, 2nd ed.; International Series of Monographs on Physics; Oxford University Press, 2013.

(4) Asbóth, L. O. J. K.; Pályi, A. A Short Course on Topological Insulators. Lecture Notes in Physics, 1st ed.; Springer, 2016; Vol. 919.

(5) Kivelson, S. Electron fractionalization,. Synth. Met. 2001, 125, 99-106.

(6) Jackiw, R.; Rebbi, C. Solitons with fermion number 1/2,. Phys. Rev. D: Part. Fields 1976, 13, 3398-3409.

(7) Sethna, J. P.; Kivelson, S. Photoinduced soliton pair production in polyacetylene: An instanton approach,. Phys. Rev. B: Condens. Matter Mater. Phys. 1982, 26, 3513-3516.

(8) Blanchet, G. B.; Fincher, C. R.; Heeger, A. J. Excitation profile for photogeneration of solitons in trans- $(\mathrm{CH})_{\mathrm{x}}$. Phys. Rev. Lett. 1983, $51,2132-2135$. 
(9) Kiess, H.; Keller, R.; Baeriswyl, D.; Harbeke, G. Photoconductivity in trans $(\mathrm{ch}) \mathrm{x}$ : A proof for the existence of solitons? Solid State Commun. 1982, 44, 1443-1447.

(10) Su, Z.-B.; Yu, L. Soliton pair generation in polyacetylene: A lattice relaxation approach,. Phys. Rev. B: Condens. Matter Mater. Phys. 1983, 27, 5199-5202.

(11) Meier, E. J.; An Fangzhao, A.; Gadway, B. Observation of the topological soliton state in the su-schrieffer-heeger model,. Nat. Commun. 2016, 7, 13986.

(12) Wang, S.; Sun, Q.; Gröning, O.; Widmer, R.; Pignedoli, C. A.; Cai, L.; Yu, X.; Yuan, B.; Li, C.; Ju, H.; Zhu, J.; Ruffieux, P.; Fasel, R.; $\mathrm{Xu}, \mathrm{W}$. On-surface synthesis and characterization of individual polyacetylene chains,. Nat. Chem. 2019, 11, 924-930.

(13) Chiang, C. K.; Fincher, C. R.; Park, Y. W.; Heeger, A. J.; Shirakawa, H.; Louis, E. J.; Gau, S. C.; MacDiarmid, A. G. Electrical conductivity in doped polyacetylene,. Phys. Rev. Lett. 1977, 39, 10981101.

(14) Lauchlan, L.; Etemad, S.; Chung, T. C.; Heeger, A. J.; MacDiarmid, A. G. Photoexcitations in polyacetylene,. Phys. Rev. B: Condens. Matter Mater. Phys. 1981, 24, 3701-3711.

(15) Kuroda, S.-I. Electron nuclear double resonance spectroscopy of solitons and polarons in conjugated polymers,. Int. J. Mod. Phys. B 1995, 09, 221-260.

(16) Su, W. P.; Schrieffer, J. R.; Heeger, A. J. Solitons in polyacetylene,. Phys. Rev. Lett. 1979, 42, 1698-1701.

(17) Boudreaux, D. S.; Chance, R. R.; Brédas, J. L.; Silbey, R. Solitons and polarons in polyacetylene: Self-consistent-field calculations of the effect of neutral and charged defects on molecular geometry,. Phys. Rev. B: Condens. Matter Mater. Phys. 1983, 28, 69276936.

(18) Patera, L. L.; Queck, F.; Scheuerer, P.; Repp, J. Mapping orbital changes upon electron transfer with tunnelling microscopy on insulators,. Nature 2019, 566, 245-248.

\section{NOTE ADDED AFTER ASAP PUBLICATION}

Due to a production error, this paper was published ASAP on March 9, 2020, with errors in equation 1. The corrected version was reposted on March 11, 2020. 